\title{
Reviving a Lost Art: Hot-water Irrigation Treatment for Intractable Epistaxis
}

\author{
Winnie Yeung ${ }^{1}$, Owen Judd ${ }^{2}$
}

\begin{abstract}
Introduction: Epistaxis is a common presentation in ear, nose, and throat (ENT). Given its substantial healthcare burden and associated morbidity, much work has been done to ascertain optimal management. Established treatment modalities include cautery and intranasal packing. Here, we describe our experience of the long forgotten yet efficacious and cost-effective technique of hot-water irrigation in patients with epistaxis. Materials and methods: Hot water is flushed briskly into the nasal cavity of an actively bleeding patient. Irrigation is continued until the water running out of the nose is clear.

Results: Intractable epistaxis has been successfully managed in two patients with elevated bleeding risk. Patient A presented with incidental thrombocytopenia. Patient B presented having been anticoagulated with low-molecular weight heparin (LMWH) for deep vein thrombosis (DVT). Active epistaxis ceased with hot-water irrigation, and both patients were discharged with no further readmissions.

Discussion: We recommend that the lost art of hot-water irrigation is an additional safe and useful tool to combat the common presentation of epistaxis.

Keywords: Anticoagulation, Deep vein thrombosis, Epistaxis, Hot water, Irrigation, Low-molecular weight heparin, Nosebleed, Thrombocytopenia. Clinical Rhinology An International Journal (2019): 10.5005/jp-journals-10013-1349
\end{abstract}

\section{INTRODUCTION}

Epistaxis is one of the most common acute presentations in ear, nose, and throat (ENT). It has been estimated that $60 \%$ of the population worldwide will experience at least one nosebleed at some point in their lives. ${ }^{1}$ Of these, $6 \%$ will present for further medical treatment. ${ }^{1}$ It accounts for over 25,000 admissions per annum in the UK alone, ${ }^{2}$ where it has been estimated that each ENT surgeon admits an average of 10.2 patients over a 3 month period for this problem, translating into an average hospital inpatient stay of 2.9 days. $^{3}$ Given its substantial burden on valuable healthcare resources and its associated morbidity for patients, much work has been undertaken to establish its optimal management, with the UK National Epistaxis Management Audit of 2016 being one such large-scale effort. ${ }^{3}$ Established mainstream treatment modalities for mild to moderate cases include nasal cautery and packing with intranasal agents. ${ }^{4,5}$ In this paper, we describe our experience of the long forgotten yet highly efficacious and cost-effective technique of hot-water nasal irrigation in two patients with intractable epistaxis.

\section{Materials and Methods}

One liter of freshly boiled water is diluted with cool tap water in a large bowl until the water temperature is cooled between 45 and $50^{\circ} \mathrm{C}$. The actively bleeding patient is positioned forward with his head leant over a sink. Hot water is flushed briskly into the patient's nasal cavity with a clean $50 \mathrm{~mL}$ syringe. The side of the bleeding is treated first. During periods of active flushing, the patient is requested to hold his breathe to prevent aspiration. The patient is encouraged to inhale deeply through his mouth in-between hot-water flushes. The water is permitted to flow freely in and out of the nasal cavities in a similar fashion to nasal douching. The hotwater flushes are continued until epistaxis has been ceased and the water running into the sink is clear. The patient is then observed for 30 minutes for signs of bleeding recurrence. ${ }^{1}$ Department of Otorhinolaryngology, Nottingham University
Hospitals NHS Trust, Nottingham, Nottinghamshire, UK
${ }^{2}$ Department of Otorhinolaryngology, University Hospitals of Derby
and Burton NHS Trust, Derby, Derbyshire, UK

Corresponding Author: Winnie Yeung, Department of Otorhinolaryngology, Nottingham University Hospitals NHS Trust, Nottingham, Nottinghamshire, UK, Phone: +44 7809119265, e-mail: winnie.yeung@nhs.net

How to cite this article: Yeung W, Judd O. Reviving a Lost Art: Hotwater Irrigation Treatment for Intractable Epistaxis. Clin Rhinol An Int J 2019;12(1):1-2.

Source of support: Royal Derby Hospital

Conflict of interest: None

\section{Results}

Intractable epistaxis has been successfully managed in two patients ( $A$ and $B$ ) with elevated bleeding risk using the method described.

\section{Patient A}

A 66-year-old male presented with left-sided epistaxis. Bleeding was initially controlled with unilateral nondissolvable intranasal packing. Incidental finding of thrombocytopenia was identified on blood tests at admission, which revealed a platelet count of $1 \times 10^{9}$ per liter. This rose to $51 \times 10^{9}$ per liter after treatment with intravenous immunoglobulin. Intranasal packing was removed after 48 hours. The patient was discharged when no further nosebleeds occurred. The patient was readmitted to hospital less than 12 hours later, presenting with recurrent epistaxis on the same side. Platelet count on readmission was $46 \times 10^{9}$ per liter. The nasal cavity was repacked with nondissolvable intranasal agent kept in situ for further 48 hours. When the repeat nasal packing was removed, the patient continued to have active moderate volume bleeding from 
the left nasal cavity. The patient was treated with hot-water nasal irrigation. Epistaxis ceased. The patient was discharged following a period of observation with no further readmissions.

\section{Patient B}

A 61-year-old male presented with left-sided epistaxis shortly after commencing low-molecular weight heparin (LMWH) anticoagulation treatment for deep vein thrombosis (DVT) of the left upper limb. He had recently completed chemoradiotherapy treatment for tonsillar cancer. Admission blood tests revealed hemoglobin of 127 and a normal clotting screen. Clinical examination with anterior rhinoscopy demonstrated a small anterior septal perforation and widespread nasal mucositis. The heparin was temporarily held. Bleeding was controlled with a nondissolvable intranasal pack, which was kept in situ for 72 hours. When the nasal packing was removed, fresh active bleeding occurred from both nasal cavities. Epistaxis stopped following treatment with hot-water nasal irrigation. The patient was discharged following observation with no further readmissions.

\section{Discussion}

The British Rhinological Society has recently published consensus recommendations on evidence-based best practice for epistaxis management following rigorous appraisal of relevant literature. ${ }^{6}$ The five management domains acknowledged included initial assessment, cautery, intranasal agents, hematological factors, and surgery/radiological intervention. However, it conceded that there is a general paucity of high-level evidence in this area, advising an element of caution be exercised when implementing their recommendations. ${ }^{6}$ The management of epistaxis, it seems, is more of an art rather than an exact science. Hot-water irrigation of the nose as a treatment for epistaxis was first recognized over 100 years ago. ${ }^{7}$ The method is still prevalent in parts of Europe, especially in Denmark where the majority of its exiguous literature originates. ${ }^{8,9}$ However, this technique seems to have been largely forgotten in other parts of the world and is not a skill routinely taught to ENT trainees in the UK.

Although not widely practiced, it was shown to be highly effective, especially in challenging posterior epistaxis, where it was successful in $82 \%$ of cases in which it was deployed. ${ }^{10,11}$ Futhermore, having been demonstrated to be at least as efficacious as other conventional methods, ${ }^{10}$ hot-water nasal irrigation has several over-reaching advantages. In addition to being a straightforward skill to perform, no expensive specialist equipment is required. A bowl of hot water and a large plastic syringe is all that is needed to carry out the technique, rendering it a comparatively cost-effective option. Existing literature advocates the use of a balloon catheter to secure the posterior choana; ${ }^{8-11}$ however, we found that this was not necessary as long as there is good communication between the doctor and the patient during the procedure.

From our own experience, patient feedback for this method has been exemplary. Although some discomfort is experienced during the procedure, all patients have found hot-water irrigation treatment more acceptable compared to the painful alternative of intranasal packing. Indeed, others have found that surgical intervention and admission to hospital in the first place has been avoided in some cases with hot-water nasal irrigation treatment. ${ }^{12}$

The physical removal of crusting, old blood, and clots with water allows a clear view of the nasal cavity for further clinical examination with anterior rhinoscopy or flexible nasendoscopy. This in turn may facilitate accurate identification of bleeding origins, enabling other treatment modalities such as nasal cautery to be more effective, even if bleeding cannot be fully controlled with hot-water irrigation.

We have found the hot-water irrigation technique to be especially helpful in patients with hematological disorders or in cases of refractory epistaxis, where this treatment may be considered as an alternative before repacking the nose to prevent further unnecessary trauma to the nasal mucosa. While hot-water irrigation does not replace other more established treatment modalities, the authors hope that by shining the spotlight on this seemingly forgotten technique, another tool can be added to the arsenal to combat the common presentation of epistaxis.

\section{Summary of Learning Points}

- Epistaxis is a commonly encountered acute ENT problem for which there is a paucity of high-level evidence to guide its management.

- Established treatments include cautery and packing with intranasal agents.

- Hot-water nasal irrigation provides a simple, efficacious yet cost-effective adjunct technique to treat epistaxis.

- It is useful in patients who have rebled following intranasal packing or those whose clotting function is mildly deranged.

- It offers a more acceptable patient experience in comparison to other more painful alternative treatments.

\section{References}

1. Viehweg TL, Roberson JB, Hudson JW. Epistaxis: diagnosis and treatment. J Oral Maxillofac Surg 2006;64(3):511-518. DOI: 10.1016/ j.joms.2005.11.031.

2. INTEGRATE (National ENT Trainee Research Network). Epistaxis 2016: National audit of management. J Laryngol Otol 2017;131(12): 1131-1141. DOI: 10.1017/S002221511700202X.

3. Kotecha B, Fowler S, Harkness $P$, et al. Management of epistaxis: a national survey. Ann R Coll Surg Eng 1996;78(5):444-446.

4. Mcleod RW, Price A, Williams RJ, et al. Intranasal cautery for the management of adult epistaxis: systematic review. J Laryngol Otol 2017;131(12):1065-1092. DOI: 10.1017/S0022215117002043.

5. Iqbal I, Jones HG, Dawe N, et al. Intranasal packs and haemostatic agents for the management of adult epistaxis: systematic review. J Laryngol Otol 2017;131(12):1065-1092. DOI: 10.1017/ S0022215117002055.

6. INTEGRATE (National ENT Trainee Research Network). The British Rhinological Society multidisciplinary consensus recommendations on the hospital management of epistaxis. J Laryngol Otol 2017;131(12):1142-1156. DOI: 10.1017/S0022215117002018.

7. Yeung W, Judd O. Hot water in epistaxis. Ind Med Gaz 1889;24(2):49.

8. Stangerup SE, Dommerby H, Lau T. Hot-water irrigation as a treatment of posterior epistaxis. Rhinology 1996;34(1):18-20.

9. Stangerup SE, Dommerby $\mathrm{H}$, Siim C, et al. New modification of hot-water irrigation in the treatment of posterior epistaxis. Arch Otolaryngol Head Neck Surg 1999;125(6):686-690. DOI: 10.1001/ archotol.125.6.686.

10. Schlegel-Wagner C, Siekmann U, Linder T. Non-invasive treatment of intractable posterior epistaxis with hot-water irrigation. Rhinology 2006;44(1):90-93.

11. Seidman MD. Hot-water irrigation in the treatment of posterior epistaxis. Arch Otolaryngol Head Neck Surg 1999;125(11):1285. DOI: 10.1001/archotol.125.11.1285.

12. Novoa E, Schlegel-Wagner C. Hot water irrigation as treatment for intractable posterior epistaxis in an out-patient setting. J Laryngol Otol 2012;126(1):58-60. DOI: 10.1017/S002221511100243X. 